\section{Full-Wave Analysis of Microwave Scattering From Short Vegetation: An Investigation on the Effect of Multiple Scattering}

\author{
Yisok Oh, Young-Mi Jang, and Kamal Sarabandi
}

\begin{abstract}
A full-wave solution for polarimetric scattering from a cluster of randomly oriented three-dimensional lossy dielectric structures above an impedance surface is presented to investigate the importance of multiple scattering. The problem is formulated using an integral equation in conjunction with the exact image representation of dyadic Green's function for the half-space problem. Then, the integral equation is solved for the induced equivalent polarization currents using the method of moments. The accuracy of the numerical code is verified using other existing numerical results and experimental observations. The model is then used to examine the effect of multiple scattering among a cluster of relatively short stems and is shown that multiple scattering significantly affects the cross-polarized backscatter whereas it has a moderate effect on the copolarized backscattering depending on the stem density.
\end{abstract}

Index Terms-Impedance surface, method of moments (MoM), multiple scattering, three-dimensional lossy dielectric structures.

\section{INTRODUCTION}

Remote sensing of crops and grassland is encountered in a number of practical applications including the study of climate, hydrology, land use, agriculture, etc. Because of their capabilities to penetrate through vegetation and all-day operation, radar and radiometer sensors operating at microwave frequencies are often used in remote sensing of vegetation and soil moisture. Because of these applications, the radar and radiometer phenomenology of terrain including rough surface, vegetation-covered surfaces, and forested environments has received significant attention over the past two decades. The literature concerning electromagnetic wave interaction with vegetation is rather extensive, and a comprehensive review of all existing approaches is beyond the scope of this paper [1]-[5].

In general, these approaches can be categorized into two general methods, namely, incoherent and coherent techniques. The basis for the formulation for incoherent techniques is radiative transfer (RT). The RT formulation is heuristic and constructed based upon the law of conservation of energy. The effect of vegetation particle size, geometry, number density, and dielectric properties is accounted for in two fundamental quantities of the RT formulation known as the phase and extinction matrices. On the other hand, coherent approaches are constructed from the wave equation and are capable of accounting for coherence effects resulting from the vegetation structures [6]-[9]. Most the coherent models are developed assuming single-scattering theory, i.e., the effect of multiple scattering is ignored. The first-order scattering approximation seems to be valid for copolarized backscatter and for conditions where the dominant scatterers in the medium are sparse.

Manuscript received May 1, 2002; revised August 8, 2002. This work was supported in part by the Agency for Defense Development of Korea and FCS Communications Program of DARPA.

Y. Oh is with the Department of Radio Science and Communication Engineering, Hong-ik University, Seoul 121-791, Korea.

Y.-M. Jang was with the Department of Radio Science and Communication Engineering, Hong-ik University, Seoul, Korea. She is now with the R\&D Center, LG Innotek, Yong-in, Korea.

K. Sarabandi is with the Department of Electrical Engineering and Computer Science, University of Michigan, Ann Arbor, MI 48109-2122 USA.

Digital Object Identifier 10.1109/TGRS.2002.805085
To predict cross-polarized backscatter correctly, higher order interparticles scattering must be taken into account. A numerical solution of RT formulation (referred to as the discrete ordinate eigenanalysis) can, in principle, account for all multiple scattering in a random medium [10]. However, there are two problems with this approach when applied to vegetation. One problem is that the RT formulation is no longer valid when the size of the constituent particles are large compared to the field correlation distance in the medium or comparable to the extent of the random medium itself. This is the case for both forested environments as well as grasslands. The second problem is that the phase matrix is constructed based on single scattering, and all near-field effects are inherently ignored.

To our knowledge, the model presented in [11] is the only approach that takes near-field second-order scattering into account. Although significant improvement in accuracy is achieved using this method, it region of validity is not known and is expected to fail for dense vegetation clusters such as a grassland or a rice field. In this paper, we consider a full-wave analysis to calculate scattering from an entire grass plant above a ground surface that obviously includes all scattering interactions of the grass blades within a given plant. Characterizing the scattering from individual plants and macromodeling the statistical behavior of its radar backscatter as a function of plant type, its moisture content, and growth stage as a building block would allow the construction of a simple and yet very accurate scattering model for grasslands.

In wetland rice cultivation, for example, a water layer over a flooded soil, or a very wet smooth soil layer in some cases, remains in fields for the growing period up to harvest season. The numerical formulation considered here in order to calculate the electromagnetic wave scattering from arbitrary-shaped vegetation elements above a flat and lossy surface is based on a volumetric integral equation whose kernel is the Green's function for a half-space impedance surface. In general, the fields of an infinitesimal dipole above a lossy half-space include calculation of Sommerfeld-type integrals, which are highly oscillatory and computationally inefficient. In the present study, a wet soil or water surface is modeled by an impedance surface, which is an accepted approximation at microwave frequencies [12], [13]. Employing an appropriate integral transform, the dyadic Green's function can be represented in terms of rapidly converging integrals [13], [14]. Then, using this formulation, the scattering from clusters of short vegetation is calculated numerically using the method of moments (MoM).

\section{FORMULATION}

The geometry of the scattering problem is shown in Fig. 1. In this problem, the interest is in the calculation of the scattered field from a collection of small plants (short vegetation) above a flat and lossy ground surface illuminated by a plane wave at microwave frequencies. As mentioned earlier, the MoM applied to a volumetric integral equation is considered for the calculation of the scattered fields. Volumetric integral equations for the polarization currents as opposed to surface integral equations for the fictitious surface currents are preferred because the scatterers are very thin compared to the wavelength. Using the volume equivalence theorem, a three-dimensional (3-D) dielectric object can be replaced by an equivalent volumetric current distribution given by

$$
\bar{J}(\bar{r})=-i k_{0} Y_{0}\left(\varepsilon_{r}(\bar{r})-1\right) \bar{E}^{t}(\bar{r})
$$

where $\bar{J}(\bar{r})$ is the equivalent volumetric (polarization) current; $\varepsilon_{r}(\bar{r})$ represents the relative dielectric constant of the object; $Y_{0}$ is the free- 


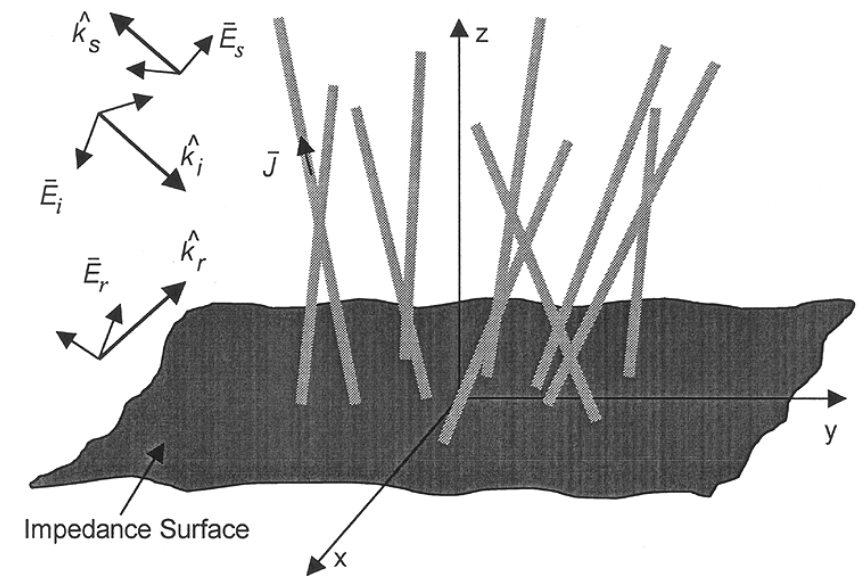

Fig. 1. Geometry for a backscatter simulation.

space admittance; and $\bar{E}^{t}(\bar{r})$ is the total field inside the scatter. This total field is composed of the incident $\left(\bar{E}^{i}(\bar{r})\right)$, the reflected $\left(\bar{E}^{r}(\bar{r})\right)$, and the scattered $\left(\bar{E}^{s}(\bar{r})\right)$ fields, i.e.,

$$
\bar{E}^{t}(\bar{r})=\bar{E}^{i}(\bar{r})+\bar{E}^{r}(\bar{r})+\bar{E}^{s}(\bar{r})
$$

where

$$
\begin{aligned}
& \bar{E}^{i}(\bar{r})=\left(E_{h}^{i} \hat{h}_{i}+E_{v}^{i} \hat{v}_{i}\right) e^{i k_{0} \hat{k}_{i} \cdot \bar{r}} \\
& \bar{E}^{r}(\bar{r})=\left(\Gamma_{h} E_{h}^{i} \hat{h}_{r}+\Gamma_{v} E_{v}^{i} \hat{v}_{r}\right) e^{i k_{0} \hat{k}_{r} \cdot \bar{r}} \\
& \bar{E}^{s}(\bar{r})=i k_{0} Z_{0} \int_{v} \overline{\bar{G}}\left(\bar{r}, \bar{r}^{\prime}\right) \cdot \bar{J}\left(\bar{r}^{\prime}\right) d v^{\prime} .
\end{aligned}
$$

Here $\hat{h}$ and $\hat{v}$ denote the unit vectors of the electric fields for $h$-polarized and $v$-polarized waves, respectively. Also, $\Gamma_{h}$ and $\Gamma_{v}$ represent the Fresnel reflection coefficients for horizontal and vertical polarizations, respectively; $Z_{0}$ is the free-space wave impedance, and $\overline{\bar{G}}\left(\bar{r}, \bar{r}^{\prime}\right)$ is the dyadic Green's function.

The dyadic Green's function for a lossy half-space medium does not have a closed form and is usually expressed in terms of Sommerfeld-type integrals, which are highly oscillatory and difficult to evaluate numerically. Here, the Green's function of an impedance surface is derived in terms of rapidly converging integrals using appropriate integral transforms. For an infinitesimal vertical electric dipole over an impedance surface, the electromagnetic field in air $(z>0)$ can be derived from a $z$-directed Hertz vector $\left(\Pi_{z}\right)$ [15]. For an infinitesimal horizontal electric dipole along the $x$ direction, an $x$-component of the Hertz vector alone cannot describe the electric field everywhere. One acceptable resolution for the $x$-directed dipole source is $\left(\Pi_{x}, 0, \Pi_{z}\right)$, which are consistent with the boundary conditions on $\bar{E}$ and $\bar{H}$ [16], [17]. The Hertz vector in air for a $q$-oriented infinitesimal current source and $p$-polarized scattered electric field can be written as $\Pi_{p q}(\bar{r})=i / \omega \varepsilon_{0}\left(G_{0}\left(\bar{r}, \bar{r}^{\prime}\right)+\tilde{G}_{p q}^{r}\left(\bar{r}, \bar{r}^{\prime}\right)\right)$. The free-space Green's function $G_{0}\left(\bar{r}, \bar{r}^{\prime}\right)$ can be expressed in a well-known spectral domain representation in terms of a continuous spectrum of plane waves. The Green's function due to the impedance surface $\tilde{G}_{p q}^{r}\left(\bar{r}, \bar{r}^{\prime}\right)$ can be expressed in terms of a continuous spectrum of plane waves containing integrals like

$$
\begin{aligned}
\tilde{G}_{p q}^{r}\left(\bar{r}, \bar{r}^{\prime}\right)=\frac{i}{8 \pi^{2}} \int_{-\infty}^{+\infty} \int_{p q}\left(k_{x}, k_{y}\right) \\
\cdot \frac{e^{i k_{x}\left(x-x^{\prime}\right)+i k_{y}\left(y-y^{\prime}\right)+i k_{z}\left(z+z^{\prime}\right)}}{k_{z}} d k_{x} d k_{y}
\end{aligned}
$$

where $k_{z}=\left(k_{0}^{2}-k_{x}^{2}-k_{y}^{2}\right)^{1 / 2}$, and $R_{p q}\left(k_{x}, k_{y}\right)$ represents the coefficient of the reflected wave for a $q$-oriented infinitesimal current source and $p$-polarized scattered electric field. The coefficient of each reflected plane wave $\left(R_{p q}\left(k_{x}, k_{y}\right)\right)$ can be obtained using the boundary conditions at the air/impedance surface interface [17]. For example, $R_{x x}\left(k_{x}, k_{y}\right)=R_{h}(\gamma)$, $R_{z x}\left(k_{x}, k_{y}\right)=2 k_{x} k_{z} / k_{0}^{2} \cdot\left(k_{z}-k_{0} \sqrt{\varepsilon_{r}}\right) /\left(\varepsilon_{r} k_{z}+k_{0} \sqrt{\varepsilon_{r}}\right)$, and $R_{z z}\left(k_{x}, k_{y}\right)=R_{v}(\gamma)$, where $R_{h}(\gamma)=(\eta \cos \gamma-1) /(\eta \cos \gamma+1)$ and $R_{v}(\gamma)=(\cos \gamma-\eta) /(\cos \gamma+\eta)$ are the horizontal and vertical reflection coefficients, respectively [12], [13]. In these equations, $\gamma$ is a complex incidence angle of each plane wave $\gamma=\arccos \left(k_{z} / k_{0}\right)$, and $\eta$ is the normalized impedance of the impedance surface $\left(\eta=Z / Z_{0}\right)$ [12], [13].

We can rewrite $R_{h}(\gamma)$ and $R_{v}(\gamma)$ as $R_{h}(\gamma)=1-2 \alpha /\left(\alpha+k_{z}\right)$ and $R_{v}(\gamma)=1-2 \beta /\left(\beta+k_{z}\right)$, respectively, where $\alpha=k_{0} / \eta$ and $\beta=k_{0} \eta$. Then, the reflection coefficients for an impedance surface can be transformed in a similar manner employed in derivation of the exact image theory [14], using the inverse Laplace transform of an exponential function, i.e.,

$$
R_{h}=1-2 \alpha \int_{0}^{\infty} e^{-\alpha \mu} e^{-k_{z} \mu} d \mu, \quad \operatorname{Re}(\alpha)>0
$$

and

$$
R_{v}=1-2 \beta \int_{0}^{\infty} e^{-\beta \mu} e^{-k z^{\mu}} d \mu, \quad \operatorname{Re}(\beta)>0 .
$$

Substituting the partial derivatives with respect to $x$ and $z$ by $i k_{x}$ and $i k_{z}$, respectively, a term like $R_{z x}\left(k_{x}, k_{y}\right)$ can be replaced by

$$
\begin{aligned}
R_{z x} & =-\frac{2}{k_{0}^{2} \varepsilon_{r}} \frac{\partial^{2}}{\partial x \partial z}\left[1-\frac{\left(1+\varepsilon_{r}\right) \beta}{\beta+k_{z}}\right] \\
& =-\frac{2}{k_{0}^{2} \varepsilon_{r}} \frac{\partial^{2}}{\partial x \partial z}\left[1-\left(1+\varepsilon_{r}\right) \beta \int_{0}^{\infty} e^{-\beta \mu} e^{-k_{z} \mu} d \mu\right] .
\end{aligned}
$$

By interchanging the order of integration and evaluating the spectral domain integration analytically over $k_{x}$ and $k_{y}$, we can obtain the Hertz vectors for three principal directions for the orientation of the infinitesimal dipoles. Consequently, the elements of the dyadic Green's function are found to be

$$
\begin{aligned}
G_{p p} & =g_{p p}+\partial^{2} g_{p p} / k_{0}^{2} \partial p^{2}+\partial^{4} g_{z p} / k_{0}^{4} \partial p^{2} \partial z^{2} \\
G_{p q} & =\partial^{2} g_{p p} / k_{0}^{2} \partial p \partial q+\partial^{4} g_{z p} / k_{0}^{4} \partial p \partial q \partial z^{2}, \quad p \neq q \\
G_{z p} & =\partial^{2} g_{p p} / k_{0}^{2} \partial p \partial z+g_{z p}+\partial^{4} g_{z p} / k_{0}^{4} \partial p \partial z^{3} \\
G_{p z} & =\partial^{2} g_{z z} / k_{0}^{2} \partial p \partial z \\
G_{z z} & =g_{z z}+\partial^{2} g_{z z} / k_{0}^{2} \partial z^{2}
\end{aligned}
$$

where

$$
\begin{aligned}
& g_{p p}\left(\bar{r}, \bar{r}^{\prime}\right)=G_{0}\left(r_{0}\right)+G_{1}\left(r_{1}\right)-2 \alpha \int_{0}^{\infty} e^{-\alpha \mu} G_{2}\left(r_{2}\right) d \mu \\
& g_{z p}\left(\bar{r}, \bar{r}^{\prime}\right)=-\frac{2}{\varepsilon_{2}} G_{1}\left(r_{1}\right)+\frac{1+\varepsilon_{r}}{\varepsilon_{r}} 2 \beta \int_{0}^{\infty} e^{-\beta \mu} G_{2}\left(r_{2}\right) d \mu \\
& g_{z z}\left(\bar{r}, \bar{r}^{\prime}\right)=G_{0}\left(r_{0}\right)+G_{1}\left(r_{1}\right)-2 \beta \int_{0}^{\infty} e^{-\beta \mu} G_{2}\left(r_{2}\right) d \mu
\end{aligned}
$$

In (6)

$$
G_{i}\left(r_{i}\right)=\frac{\exp \left[i k_{0} r_{i}\right]}{4 \pi r_{i}}, \quad i=0,1,2
$$

where

$$
\begin{aligned}
p, q & =x, y \quad r_{0}=\sqrt{\left(x-x^{\prime}\right)^{2}+\left(y-y^{\prime}\right)^{2}+\left(z-z^{\prime}\right)^{2}} \\
r_{1} & =\sqrt{\left(x-x^{\prime}\right)^{2}+\left(y-y^{\prime}\right)^{2}+\left(z+z^{\prime}\right)^{2}}
\end{aligned}
$$


and

$$
r_{2}=\sqrt{\left(x-x^{\prime}\right)^{2}+\left(y-y^{\prime}\right)^{2}+\left(z+z^{\prime}+i \mu\right)^{2}} .
$$

This formulation is exact for an impedance surface independent of source or observation point positions. The last term of (6) includes an integral over $0<\mu<\infty$ whose integrand can be interpreted as a linear distributed image source in the complex $z$ plane along the imaginary axis at the mirror image point $\left(-z^{\prime}-i \infty<z<-z^{\prime}\right)$. It should be noted that the integrals converge rapidly due to exponential decays of the integrands [13].

\section{MOM SOLUTION}

The integral equation given by (2) can be solved numerically using the MoM. Among many other bases functions, we chose the volumetric brick as the basis function in the limit of electrically small subdomains [18] for simplicity. Using the point-matching technique, the integral equation can be cast into the following matrix equation:

$$
\left[\begin{array}{lll}
{\left[Z_{m n}^{x x}\right]} & {\left[Z_{m n}^{x y}\right]} & {\left[Z_{m n}^{x z}\right]} \\
{\left[Z_{m n}^{y x}\right]} & {\left[Z_{m n}^{y y}\right]} & {\left[Z_{m n}^{y z}\right]} \\
{\left[Z_{m n}^{z x}\right]} & {\left[Z_{m n}^{z y}\right]} & {\left[Z_{m n}^{z z}\right]}
\end{array}\right]\left[\begin{array}{l}
{\left[I_{n}^{x}\right]} \\
{\left[I_{n}^{y}\right]} \\
{\left[I_{n}^{z}\right]}
\end{array}\right]=\left[\begin{array}{l}
{\left[V_{m}^{x}\right]} \\
{\left[V_{m}^{y}\right]} \\
{\left[V_{m}^{z}\right]}
\end{array}\right]
$$

where $I_{n}^{p}$ is an unknown constant of the $n$th basis function for the $p$-component of the volume currents $(p=x, y, z)$

$$
\begin{aligned}
& V_{m}^{p}=-i k_{0} Y_{0}\left[\varepsilon_{r}\left(\bar{r}_{m}\right)-1\right] \\
& \cdot\left(\hat{e}_{i} e^{i k_{0} \hat{k}_{i} \cdot \bar{r}_{m}}+\Gamma_{e} \hat{e}_{r} e^{i k_{0} \hat{k}_{r} \cdot \bar{r}_{m}}\right) \cdot \hat{p}, \quad e=h, v ; p=x, y, z
\end{aligned}
$$$$
Z_{m n}^{p q}=\delta_{p q} \delta_{m n}-k_{0}^{2}\left[\varepsilon_{r}\left(\bar{r}_{m}\right)-1\right]
$$$$
\cdot \int_{\Delta V_{n}} G_{p q}\left(\bar{r}_{m}, \bar{r}_{n}\right) d v_{n}, \quad p, q=x, y, z .
$$

In (10), $\delta_{p q}$ and $\delta_{m n}$ are the Kronecker delta functions, and $\bar{r}_{m}$ and $\bar{r}_{n}$ represent the $m$ th matching position (observation point) and the $n$th integration position, respectively. Explicit form of differentiations of the Green's functions in (5)-(7) are given by

$$
\begin{aligned}
\partial^{2} G_{i} / \partial p^{2}= & G_{i}\left(r_{i}\right)\left\{p_{m n}^{2} f_{1} / r_{i}^{4}-\left(1-i k_{0} r_{i}\right) / r_{i}^{2}\right\} \\
\partial^{2} G_{i} / \partial z^{2}= & G_{i}\left(r_{i}\right)\left\{z_{i m n}^{2} f_{1} / r_{i}^{4}-\left(1-i k_{0} r_{i}\right) / r_{i}^{2}\right\} \\
\partial^{2} G_{i} / \partial p \partial q= & G_{i}\left(r_{i}\right) p_{m n} q_{m n} f_{1} / r_{i}^{4}, \quad p \neq q \\
\partial^{2} G_{i} / \partial p \partial z= & G_{i}\left(r_{i}\right) p_{m n} z_{i m n} f_{1} / r_{i}^{4} \\
\partial^{4} G_{i} / \partial p^{2} \partial z^{2}= & G_{i}\left(r_{i}\right)\left\{f_{1} / r_{i}^{4}+\left(p_{m n}^{2}+z_{i m n}^{2}\right)\right. \\
& \left.\cdot f_{2} / r_{i}^{6}+p_{m n}^{2} z_{i m n}^{2} f_{3} / r_{i}^{8}\right\} \\
\partial^{4} G_{i} / \partial x \partial y \partial z^{2}= & G_{i}\left(r_{i}\right) x_{m n} y_{m n}\left\{f_{2} / r_{i}^{6}+z_{i m n}^{2} f_{3} / r_{i}^{8}\right\} \\
\partial^{4} G_{i} / \partial p \partial z^{3}= & G_{i}\left(r_{i}\right) p_{m n} z_{i m n}\left\{3 f_{2} / r_{i}^{6}+z_{i m n}^{2} f_{3} / r_{i}^{8}\right\}
\end{aligned}
$$

where

$$
\begin{aligned}
p, q & =x, y \quad i=0,1,2 \quad p_{m n} \equiv p_{m}-p_{n} \\
q_{m n} & \equiv q_{m}-q_{n} \quad z_{1 m n} \equiv z_{m}-z_{n} \quad z_{2 m n} \equiv z_{m}+z_{n} \\
z_{3 m n} & \equiv z_{m}+z_{n}+i \mu \quad f_{1}=3-i 3 k_{0} r_{i}-k_{0}^{2} r_{i}^{2} \\
f_{2} & =-15+i 15 k_{0} r_{i}+6 k_{0}^{2} r_{i}^{2}-i k_{0}^{3} r_{i}^{3} \\
f_{3} & =105-i 105 k_{0} r_{i}-45 k_{0}^{2} r_{i}^{2}+i 10 k_{0}^{3} r_{i}^{3}+k_{0}^{4} r_{i}^{4} .
\end{aligned}
$$

$G_{i}$ and $r_{i}$ are given in (7).

When $n=m$, i.e., for the case of self-cell integration, the second derivatives of the free-space Green's function $G_{0}\left(r_{0}\right)$ produce the wellknown singularity that is not integrable. It should be noted that this is an artifact of interchanging the order of differentiations and integrations going from the vector potentials to the dyadic Green's function. There

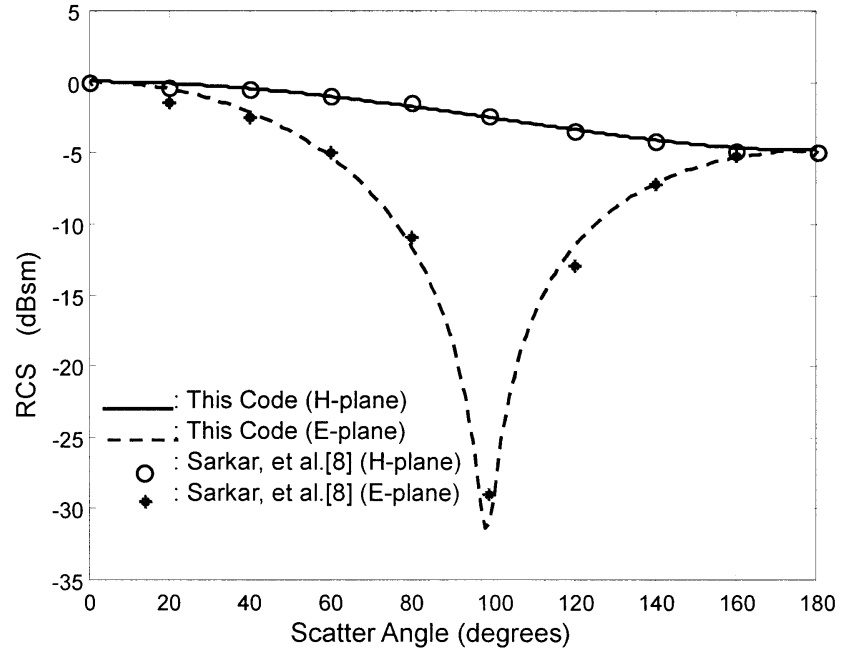

Fig. 2. RCS of a dielectric cube $\left(a=\lambda / 5, \varepsilon_{r}=9\right)$ in free space.

are well-known techniques to remove this singularity and express the integrals in terms of their principal values [19]. This procedure is

$$
\begin{aligned}
\int_{\Delta V_{n}} \frac{\partial^{2}}{\partial p \partial q} G_{0} d v=\int_{\Delta V_{n}-V_{\varepsilon}} \frac{\partial^{2}}{\partial p \partial q} G_{0} d v \\
+\int_{V_{\varepsilon}} \frac{\partial^{2}}{\partial p \partial q}\left(G_{0}-g_{0}\right) d v+\int_{V_{\varepsilon}} \frac{\partial^{2}}{\partial p \partial q} g_{0} d v
\end{aligned}
$$

where $V_{\varepsilon}$ is a small finite volume surrounding the observation point, and $g_{0} \equiv 1 / 4 \pi r_{0}$. We can evaluate the second and the third integrals in (12) explicitly for a small sphere centered at the observation point with radius $a$, which leads to $\delta_{p q}\left\{1-(1-i k a) e^{i k a}\right\} / 3$ and $\delta_{p q}(-1 / 3)$, respectively. The integral of the free-space Green's function can also be explicitly evaluated over the small sphere and becomes $\{-1+(1-$ $\left.i k a) e^{i k a}\right\} / k_{0}^{2}$. Therefore, the dyadic Green's function involving the free-space scalar Green's function can be represented by

$$
\begin{aligned}
\int_{\Delta V_{n}} L_{p p} G_{0} d v_{n}=\int_{\Delta V_{n}-V_{\varepsilon}} & L_{p p} G_{0} d v_{n} \\
+ & \left\{-1+2(1-i k a) e^{i k a} / 3\right\} / k_{0}^{2}
\end{aligned}
$$

where $p=x, y, z ; L_{p p}$ is the differential operator of $\left(1+\partial^{2} / k_{0}^{2} \partial p^{2}\right)$, and the first integral can be evaluated numerically.

Once the elements of the impedance matrix and the excitation vector are calculated, the unknown equivalent volume current inside the dielectric body can be found by inverting (8). Consequently, the scattered field can be obtained from

$$
\begin{aligned}
E_{p q}^{s}=\frac{e^{i k r}}{r} \frac{i k_{0} Z_{0}}{4 \pi} & \sum_{n=1}^{N}\left\{\bar{J}_{q n}\left(\bar{r}_{n}\right) \cdot \hat{p}_{s} e^{-i k_{0} \hat{k}_{s} \cdot \bar{r}_{n}}\right. \\
& \left.+\Gamma_{p}\left(\theta_{r}\right) \bar{J}_{q n}\left(\bar{r}_{n}\right) \cdot \hat{p}_{s r} e^{-i k_{0} \hat{k}_{s r} \cdot \bar{r}_{n}}\right\} \Delta v_{n}
\end{aligned}
$$

where $p, q=h, v$.

\section{NUMERICAL RESULTS}

In this section, we present several examples to demonstrate the accuracy and capability of the full-wave solution. The first two examples pertain to verification of the numerical code by comparing the results with those obtained from other codes and measurements. First, we considered scattering from a dielectric in free space and compare the bistatic scattering radar cross section (RCS) with those reported in [20]. The dielectric cube has an edge length of $a=\lambda / 5$ and a relative dielectric constant of $\varepsilon_{r}=9$. Fig. 2 shows the comparison of the $E$ 


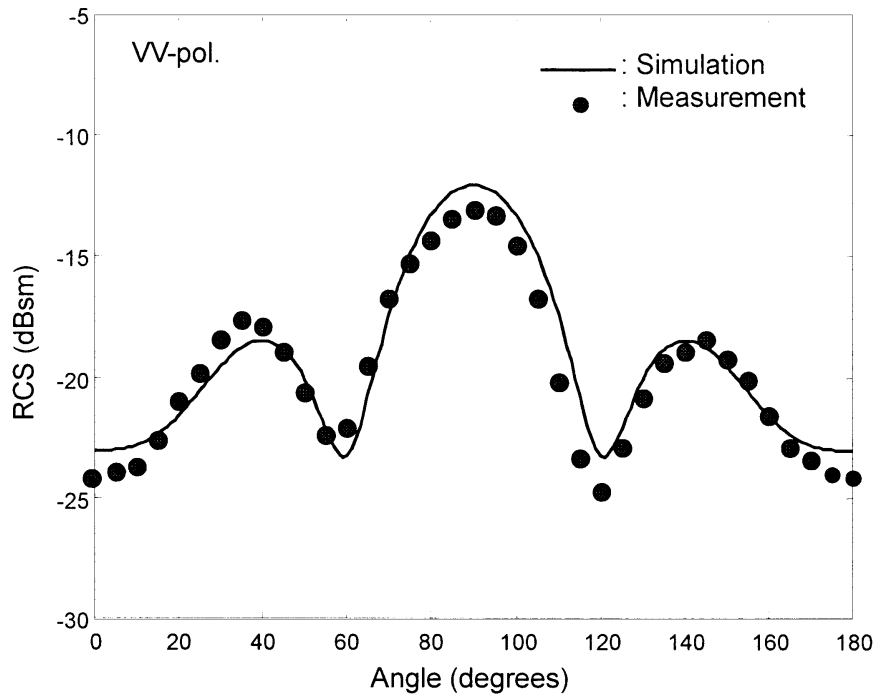

Fig. 3. RCS of a lossy dielectric box $\left(2.25 \lambda \times 1.8 \lambda \times 0.45 \lambda, \varepsilon_{r}=55.4+i 35\right)$ above a conducting surface.

and $H$ planes bistatic RCS patterns (those obtained from our codes and the results obtained from [20]) of the cube for an incident wave normal to one of the cube faces. As can be seen in Fig. 2, the bistatic RCS patterns are in excellent agreement.

To further examining the accuracy of the code, comparison of simulated results are made with the backscatter measurements of a lossy dielectric body above a conducting plane $(\eta \rightarrow 0)$. The measurements were conducted using a scatterometer, which consists of a vector network analyzer, a Ku-band antenna set, an automatic antenna positioner, and a target positioner. The scatterometer is calibrated employing the single-target calibration technique using a conducting sphere [21]. Measurements were conducted at a center frequency of $13.5 \mathrm{GHz}$. The target was a thin plastic rectangular box $(2.25 \lambda \times 1.8 \lambda \times 0.45 \lambda)$ filled with distilled water $\left(\varepsilon_{r}=5.5 .4+i 35\right.$ at $13.5 \mathrm{GHz}$ [22]). The plastic box had wall thickness of $0.02 \lambda$ and a dielectric constant of $\varepsilon_{r}=2.2$. The target was placed on an aluminum plate of $54 \lambda \times 54 \lambda$, which was large enough to include the antenna's footprint. The backscatter measurements of the target above the aluminum plate were collected at an incidence angle of $\theta=30^{\circ}$ for an azimuth angular range of $\phi=0^{\circ} \sim 180^{\circ}$ in steps of $4^{\circ}$. Considering the target and the antenna positioning errors, Fig. 3 shows good agreement between the measurement and the numerical simulation.

Then, we use this full-wave method to compute the backscattering coefficient of a collection of randomly oriented grass stems above a water surface, similar to what is shown in Fig. 1. A stem end position $(r, \varphi)$ is assumed to be uniformly distributed inside a circle with diameter $d=2 \lambda$ on the surface. The stem orientation was also assumed to be uniformly distributed in an angular range $0 \leq \theta \leq \theta_{\max }, 0 \leq$ $\phi \leq 2 \pi$. In this simulation we chose $\theta_{\max }=30^{\circ}$. The mean value of the stem length is set to $2 \lambda$ and its standard deviation to $0.3 \lambda$. All stems were given a fixed diameter of $0.113 \lambda$ and a dielectric constant of $\varepsilon_{r}=22.1+i 10.1$ corresponding to a gravimetric moisture content of $0.7 \mathrm{~g} / \mathrm{cm}^{3}$. This dielectric constant was computed according to formulas provided in [23] for vegetation material. The backscattering coefficient (the second moment of the backscatter RCS per unit area) fields were computed using a Monte Carlo simulation by generating 30 independent samples of the stem cluster. Fig. 4 shows a comparison between the full-wave method and the single-scattering method for the co- and cross-polarized backscattering coefficients. The single-scattering method ignores the effect of multiple scattering. For the single-scattering computation, the volume current distribution of

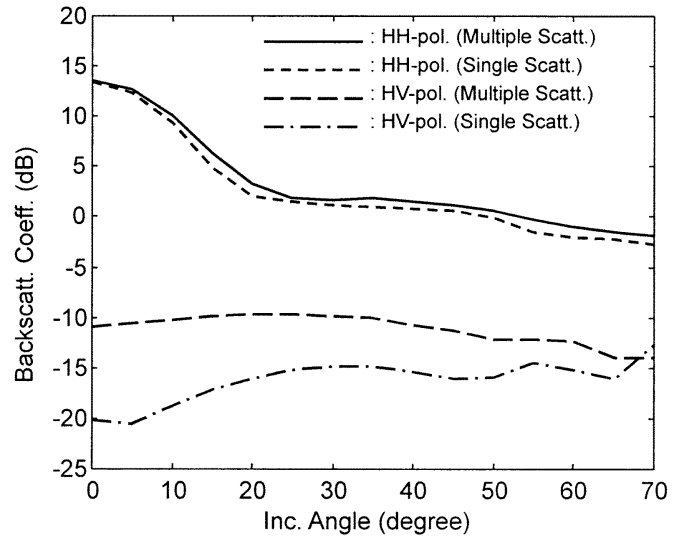

Fig. 4. Backscattering coefficients for a simple vegetation canopy, ten stems $(2 \lambda)$ in a $2 \lambda$-diameter circle above a water surface.

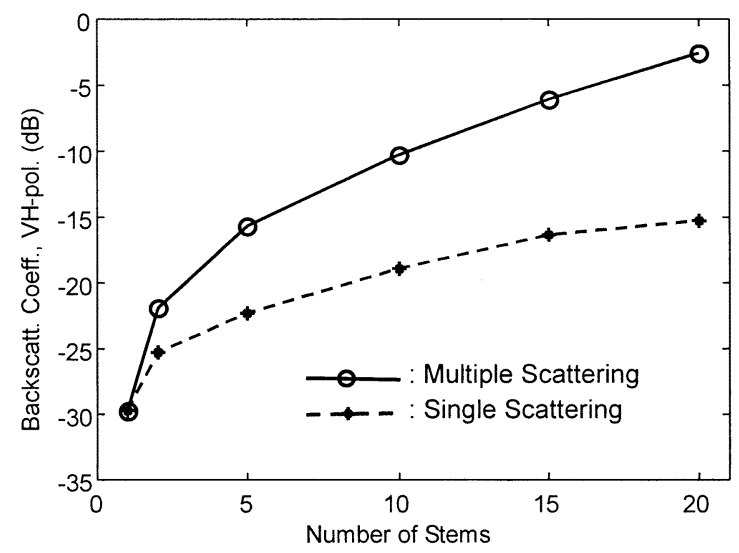

Fig. 5. Cross-polarized backscattering coefficients at $10^{\circ}$, compared with those of the single-scattering method.

each stem was computed assuming that a single stem exists above the water surface, and then the scattered fields were computed from the all ten stems in the cluster coherently. As can be seen in Fig. 4, the effect of multiple scattering for copolarized terms is negligible for this relatively sparse cluster, whereas for the cross-polarized term the effect is significant. For example, the difference between the single-scattering solution and full-wave solution for $\sigma_{v h}^{0}$ at $10^{\circ}$ is as high as $9 \mathrm{~dB}$.

In Fig. 5, we compare the cross-polarized backscattering coefficients obtained from the full-wave method with those calculated using the single scattering method as a function of stem number density. In these simulations, the incidence angle is chosen to be $10^{\circ}$ and the number of the stems in the $2 \lambda$-circle varying from 1-20. The cross-polarized backscattering coefficients are identical for a single stem as expected, but as shown the discrepancy between the single scattering results and full-wave results increases as the number of stems are increased. The discrepancy ranges from $3.5 \mathrm{~dB}$ for two stems to about $13 \mathrm{~dB}$ for 20 stems, as shown in Fig. 5.

\section{CONCLUSION}

The issue of multiple scattering effect in radar backscatter from short vegetation is examined. An efficient numerical method is developed to compute the scattering behavior of 3-D inhomogeneous dielectric structures above impedance surfaces using an appropriate integral transformation. The accuracy of this full-wave method is verified with other numerical solutions and an experimental observation. Then, this method is applied to compute the backscattering coefficients of 
a cluster of short stems. It is shown that the effect of multiple scattering on the copolarized backscattering coefficients is negligible for relatively sparse conditions, whereas the cross-polarized backscatter is significantly influenced by the multiple-scattering effect.

\section{ACKNOWLEDGMENT}

The authors are grateful to the anonymous reviewers for their valuable comments and reviews of this manuscript. The authors also thank I.-S. Koh (University of Michigan) for his valuable suggestions.

\section{REFERENCES}

[1] R. H. Lang and J. S. Sidhu, "Electromagnetic backscattering from a layer of vegetation: A discrete approach," IEEE Trans. Geosci. Remote Sensing, vol. GE-21, pp. 62-71, Jan. 1983.

[2] M. A. Karam and A. K. Fung, "Electromagnetic scattering from a layer of finite length, randomly oriented, dielectric circular cylinders over a rough interface with application to vegetation," Int. J. Remote Sens., vol. 9, pp. 1109-1134, 1988.

[3] F. T. Ulaby, K. Sarabandi, K. McDonald, M. Whitt, and M. C. Dobson, "Michigan microwave canopy scattering model," Int. J. Remote Sens., vol. 11, no. 7, pp. 1223-1253, 1990.

[4] K. Sarabandi and P. F. Polatin, "Electromagnetic scattering from two adjacent objects," IEEE Trans. Antennas Propagat., vol. 42, pp. 510-517, Apr. 1994.

[5] G. Zhang, L. Tsang, and Z. Chen, "Collective scattering effects of trees generated by stochastic Lindenmayer systems," Microwave Opt. Tech. Lett., vol. 11, pp. 107-111, Feb. 1996.

[6] S. H. Yueh, J. A. Kong, J. K. Jao, R. T. Shin, and T. L. Toan, "Branching model for vegetation," IEEE Trans. Geosci. Remote Sensing, vol. 30, pp. 390-402, Mar. 1992.

[7] J. M. Stiles, K. Sarabandi, and F. T. Ulaby, "Electromagnetic scattering from grassland: Part I, A fully coherent scattering model," IEEE Trans. Geosci. Remote Sensing, vol. 38, pp. 339-348, Jan. 2000.

[8] — - "Electromagnetic scattering from grassland: Part II, Measurement and modeling results," IEEE Trans. Geosci. Remote Sensing, vol. 38, pp. 349-356, Jan. 2000.
[9] Y. C. Lin and K. Sarabandi, "A Monte Carlo coherent scattering model for forest canopies using fractal-generated trees," IEEE Trans. Geosci. Remote Sensing, vol. 37, pp. 440-451, Jan. 1999.

[10] L. Tsang, J. A. Kong, and R. T. Shin, Theory of Microwave Remote Sensing. New York: Wiley, 1985.

[11] T. C. Chiu and K. Sarabandi, "Electromagnetic scattering from short branching vegetation," IEEE Trans. Geosci. Remote Sensing, vol. 38, pp. 911-925, Mar. 2000.

[12] T. B. A. Senior and J. L. Vloakis, Approximate Boundary Conditions in Electromagnetics. London, U.K.: IEE, 1995.

[13] K. Sarabandi, "Scattering from dielectric structures above impedance surfaces and resistive sheets," IEEE Trans. Antennas Propagat., vol. 40, pp. 67-78, Jan. 1992.

[14] I. V. Lindell and A. Alanen, "Exact image theory for the Sommerfeld half-space problem, Part III: General formulatin," IEEE Trans. Antennas Propagat., vol. 32, pp. 1027-1032, 1984.

[15] J. R. Wait, Electromagnetic Waves in Stratified Media. Piscataway, NJ: IEEE Press, 1996.

[16] A. Erteza and B. K. Park, "Nonuniqueness of resolution of Hertz vector in presence of a boundary, and the horizontal dipole problem," IEEE Trans. Antennas Propagat., vol. AP-17, pp. 376-378, May 1969.

[17] A. Mohsen, "On the evaluation of Sommerfeld integrals," Proc. Inst. Elect. Eng. H, vol. 129, no. 4, pp. 177-182, Aug. 1982.

[18] A. W. Glisson, S. M. Rao, and D. R. Wilton, "Physically-based approximation of electromagnetic field quantities," in Proc. IEEE Antenna Propagat. Soc. Int. Symp., vol. 1, June 2002, pp. 78-81.

[19] S.-W. Lee, J. Boersma, C.-L. Law, and G. A. Deschamps, "Singularity in Green's function and its numerical evaluation," IEEE Trans. Antennas Propagat., vol. AP-28, pp. 311-317, May 1980.

[20] T. K. Sarkar, E. Arvas, and S. Ponnapalli, "Electromagnetic scattering from dielectric bodies," IEEE Trans. Antennas Propagat., vol. 37, pp. 673-676, May 1989.

[21] K. Sarabandi and F. T. Ulaby, "A convenient technique for polarimetric calibration of radar system," IEEE Trans. Geosci. Remote Sensing, vol. 28, pp. 1022-1033, Nov. 1990.

[22] F. T. Ulaby, R. K. Moore, and A. K. Fung, Microwave Remote Sensing, Active and Passive. Norwood, MA: Artech House, 1986, vol. III.

[23] F. T. Ulaby and M. A. El-Rayes, "Microwave dielectric spectrum of vegetation-Part II: Dual-dispersion model," IEEE Trans. Geosci. Remote Sensing, vol. GE-25, pp. 550-557, 1987. 